\title{
Elevated preoperative hemoglobin A1c level is predictive of adverse events after coronary artery bypass surgery
}

\author{
Michael E. Halkos, MD, ${ }^{a}$ John D. Puskas, MD, ${ }^{\mathrm{b}}$ Omar M. Lattouf, MD, PhD, ${ }^{\text {a }}$ Patrick Kilgo, $\mathrm{MSc}^{\mathrm{b}}{ }^{\mathrm{b}}$ Faraz Kerendi, $\mathrm{MD}$,
} Howard K. Song, MD, PhD, ${ }^{c}$ Robert A. Guyton, MD, and Vinod H. Thourani, MD ${ }^{a}$

From the Clinical Research Unit, Division of Cardiothoracic Surgery, Emory University School of Medicine, ${ }^{a}$ Atlanta, Georgia; Department of Biostatistics, Rollins School of Public Health, Emory University School of Medicine, ${ }^{\mathrm{b}}$ Atlanta, Georgia; Division of Cardiothoracic Surgery, Oregon Health and Science University, ${ }^{\mathrm{c}}$ Portland, Oregon.

Presented at the 87th Annual Meeting of The American Association for Thoracic Surgery. Washington, DC, May 5-9, 2007.

Received for publication May 8, 2007; revisions received Jan 12, 2008; accepted for publication Feb 7, 2008.

Address for reprints: Vinod H. Thourani, MD, 550 Peachtree Street, Crawford Long Hospital, 6th Floor, Medical Office Tower, Cardiothoracic Surgery, Atlanta, GA 30308 (E-mail: vinod.thourani@emoryhealthcare. org).

J Thorac Cardiovasc Surg 2008;136:631-40 0022-5223/\$34.00

Copyright $\odot 2008$ by The American Association for Thoracic Surgery

doi:10.1016/j.jtcvs.2008.02.091
Objective: Diabetes mellitus has been associated with an increased risk of adverse outcomes after coronary artery bypass grafting. Hemoglobin A1c is a reliable measure of long-term glucose control. It is unknown whether adequacy of diabetic control, measured by hemoglobin A1c, is a predictor of adverse outcomes after coronary artery bypass grafting.

Methods: Of 3555 consecutive patients who underwent primary, elective coronary artery bypass grafting at a single academic center from April 1, 2002, to June 30, 2006, $3089(86.9 \%)$ had preoperative hemoglobin A1c levels obtained and entered prospectively into a computerized database. All patients were treated with a perioperative intravenous insulin protocol. A multivariable logistic regression model was used to determine whether hemoglobin A1c, as a continuous variable, was associated with in-hospital mortality, renal failure, cerebrovascular accident, myocardial infarction, and deep sternal wound infection after coronary artery bypass grafting. Receiver operating characteristic curve analysis identified the hemoglobin A1c value that maximally discriminated outcome dichotomies.

Results: In-hospital mortality for all patients was 1.0\% (31/3089). An elevated hemoglobin A1c level predicted in-hospital mortality after coronary artery bypass grafting (odds ratio 1.40 per unit increase, $P=.019$ ). Receiver operating characteristic curve analysis revealed that hemoglobin A1c greater than $8.6 \%$ was associated with a 4-fold increase in mortality. For each unit increase in hemoglobin A1c, there was a significantly increased risk of myocardial infarction and deep sternal wound infection. By using receiver operating characteristic value thresholds, renal failure (threshold 6.7, odds ratio 2.1), cerebrovascular accident (threshold 7.6, odds ratio 2.24), and deep sternal wound infection (threshold 7.8, odds ratio 5.29) occurred more commonly in patients with elevated hemoglobin A1c.

Conclusion: Elevated hemoglobin A1c level was strongly associated with adverse events after coronary artery bypass grafting. Preoperative hemoglobin A1c testing may allow for more accurate risk stratification in patients undergoing coronary artery bypass grafting.

$\mathrm{D}$ iabetes mellitus has long been recognized as an independent risk factor for the development of coronary artery disease. ${ }^{1}$ Although the literature is replete with studies in patients undergoing coronary artery bypass grafting (CABG) comparing outcomes in diabetic patients versus nondiabetic patients, ${ }^{2-9}$ the impact of preoperative glucose control on outcomes has not been extensively studied. ${ }^{10}$ Previous studies have used methods of glucose control (ie, diet vs oral hypoglycemics vs insulin) as a means to stratify outcomes. ${ }^{2,3,7}$ Moreover, because a spot preoperative glucose level is highly variable, it is unclear whether these treatment strategies were effective in achieving acceptable diabetes control before CABG. This is in contrast with perioperative glucose control, which has a significantly favorable impact on postoperative outcomes. ${ }^{10-15}$ 


\section{Abbreviations and Acronyms}

AUROC $=$ area under the receiver operating characteristic

$\mathrm{CABG}=$ coronary artery bypass grafting

$\mathrm{CPB}=$ cardiopulmonary bypass

CVA = cerebrovascular accident

DSWI = deep sternal wound infection

$\mathrm{HbA} 1 \mathrm{C}=$ hemoglobin A1c

LOS = length of stay

MI $=$ myocardial infarction

OR $=$ odds ratio

The American Diabetes Association has recommended the use of the blood hemoglobin A1c (HbA1c) level as a method of assessing long-term glycemic control in diabetic patients. ${ }^{16} \mathrm{HbA1c}$, also known as glycosylated hemoglobin, indicates a patient's blood glucose control during the previous 3 to 4 months. HbAlc is formed when glucose in the blood binds irreversibly to hemoglobin to form a stable glycated hemoglobin complex. Because the normal life span of red blood cells is 90 to 120 days, HbA1c will only be eliminated when red cells are replaced. Because red cell turnover is continuous and $\mathrm{HbA} 1 \mathrm{c}$ is not affected by short-term glycemic lability, HbA1c allows better assessment of glucose control during a 3- to 4-month time period. The American Diabetes Association currently recommends that patients with diabetes achieve HbA1c levels less than 7\%, which is associated with a lower risk of diabetes-associated complications. ${ }^{17,18}$

We previously reported the detrimental effects of diabetes on the outcomes after CABG; however, that study did not take into account the influence of preoperative blood glucose control. $^{2}$ The current study was performed to more specifically evaluate the role of preoperative blood glucose control (using the surrogate $\mathrm{HbA1c}$ laboratory value) on in-hospital outcomes after primary, elective, isolated CABG. Therefore, the purpose of this study was to 1) assess whether elevated $\mathrm{HbA} 1 \mathrm{c}$ can be used as a risk factor for morbidity and mortality after $\mathrm{CABG}$; 2) determine whether patients with well-controlled diabetes preoperatively $(\mathrm{HbA} 1 \mathrm{c}<7 \%)$ had better outcomes compared with patients with poorly controlled diabetes preoperatively ( $\mathrm{HbA} 1 \mathrm{c} \geq 7 \%)$; 3) determine whether patients with well-controlled diabetes $(\mathrm{HbA} 1 \mathrm{c}<7 \%)$ have outcomes comparable to those without a history of diabetes; and 4) validate the American Diabetes Association recommended $\mathrm{HbA} 1 \mathrm{c}$ thresholds and provide thresholds for previously unstudied adverse outcomes after CABG.

\section{Materials and Methods}

In compliance with Health Insurance Portability and Accountability Act of 1996 regulations and the Declaration of Helsinki, and after institutional review board approval granted by Emory University, the Society of Thoracic Surgeons Adult Cardiac Database was que- ried for all patients who underwent isolated, elective, primary CABG at Emory University Hospital, Emory Crawford Long Hospital, and Wellstar Kennestone Hospital from April 1, 2002, to June 30,2006 . This period of study coincides with the entire period during which preoperative $\mathrm{HbA} 1 \mathrm{c}$ levels were routinely collected in patients scheduled for cardiac surgery. All data for consecutive patients were prospectively entered into a computerized cardiac surgical database, using the data fields and definitions of the Society of Thoracic Surgeons National Adult Cardiac Database. Data were managed by local database staff and warehoused in locked, secure facilities, protected by computer passwords and firewalls.

Cardiopulmonary bypass (CPB) was used according to the discretion of the attending surgeon. Conventional $\mathrm{CABG}$ with $\mathrm{CPB}$ was performed with standard techniques, using roller head pumps, membrane oxygenators, cardiotomy suction, arterial filters, cold antegrade or retrograde blood cardioplegia, and moderate systemic hypothermia $\left(32^{\circ} \mathrm{C}-34^{\circ} \mathrm{C}\right)$. Off-pump $\mathrm{CABG}$ was performed with one of several commercially available cardiac positioning and coronary artery stabilizing devices. Patients who were converted intraoperatively from off-pump CABG to conventional CABG were entered into the database and analyzed according to the operation they ultimately received.

All patients were treated with a uniform perioperative intravenous insulin protocol. In the operating room, an insulin infusion was premixed with 125 units of insulin in $250 \mathrm{~mL} 0.9 \%$ normal saline. Routine measurement of blood glucose was obtained from serial arterial blood gases measured every 30 minutes. In the intensive care unit, glucose levels were obtained from arterial blood gas samples or finger stick samples every 2 hours. Once patients were transferred to the floor, blood glucose values were obtained every 4 to 6 hours. Glucose levels were obtained via arterial samples in the operating room and the intensive care unit and via finger stick blood samples on the floor. The insulin infusion was initiated for blood glucose more than $120 \mathrm{mg} / \mathrm{dL}$ and adjusted to target intraoperative blood glucose between 80 and $110 \mathrm{mg} / \mathrm{dL}$ according to the discretion of the attending cardiac anesthesiologist. In the intensive care unit, patients received a continuous insulin infusion that was adjusted to maintain blood glucose between 80 and $110 \mathrm{mg} / \mathrm{dL}$ according to a sliding scale (blood glucose $-60 \times 0.04=$ units of insulin per hour). On transfer out of the intensive care unit, most patients are monitored with blood glucose monitoring every 4 to 6 hours (goal blood glucose $<150$ $\mathrm{mg} / \mathrm{dL}$ ) and maintained according to the sliding scale with subcutaneous insulin in addition to their preoperative subcutaneous regimens. If necessary to achieve glucose control, continuous insulin infusion was continued after transfer out of the intensive care unit. Patients with newly diagnosed or poorly controlled diabetes also received endocrinology consultation for better control in the postoperative period. To adjust for the effect of perioperative glucose, 2 different mean glucose values were computed and modeled: the mean glucose value for the day of surgery and the mean glucose value for postoperative days 1 to 3 . The blood glucose level was used as a continuous variable in the multivariable analysis.

Analytically, this study aimed to determine whether $\mathrm{HbA} 1 \mathrm{c}$ was an independent risk factor of adverse outcomes. To this end, a total of 22 covariates were collected to use as risk adjustors to ensure that the effect of $\mathrm{HbA} 1 \mathrm{c}$ was not confounded by their influence. These covariates are listed in Table 1 by HbAlc groupings $(<7 \%$ and $\geq 7 \%)$. The HbA1c classifications were chosen according to recommendations 


\section{TABLE 1. Preoperative demographics and clinical variables by HbA1c risk group}

\begin{tabular}{|c|c|c|c|}
\hline \multirow[b]{2}{*}{ Risk factor } & \multirow{2}{*}{$\frac{\mathrm{HbA1c}<7.0 \%}{\mathrm{~N}=2275(\%)}$} & \multirow{2}{*}{$\frac{\mathrm{HbA1c} \geq 7.0 \%}{\mathrm{~N}=814(\%)}$} & \multirow[b]{2}{*}{$P$ value } \\
\hline & & & \\
\hline Diabetes mellitus & $516(22.7 \%)$ & $724(88.9 \%)$ & $<.001$ \\
\hline Age (mean $\pm S D$ ) & $63.0 \pm 10.9$ & $61.6 \pm 10.0$ & $<.001$ \\
\hline Female & $581(25.5 \%)$ & $265(32.6 \%)$ & $<.001$ \\
\hline Caucasian & $1871(84.2 \%)$ & $596(76.0 \%)$ & $<.001$ \\
\hline Renal failure & $104(4.6 \%)$ & $75(9.2 \%)$ & $<.001$ \\
\hline Stroke & $198(8.7 \%)$ & $91(11.2 \%)$ & .037 \\
\hline NYHA class IV & $446(19.8 \%)$ & $172(21.4 \%)$ & .341 \\
\hline CCS class & $401(17.9 \%)$ & $131(16.3 \%)$ & .319 \\
\hline Hypertension & $1783(78.4 \%)$ & $718(88.2 \%)$ & $<.001$ \\
\hline Left main disease & $505(22.2 \%)$ & $153(18.8 \%)$ & .042 \\
\hline $\begin{array}{l}\text { No. diseased vessels } \\
\quad(\text { mean } \pm S D)\end{array}$ & $3.55 \pm 0.64$ & $3.64 \pm 0.58$ & $<.001$ \\
\hline Chronic lung disease & $304(13.4 \%)$ & $93(11.4 \%)$ & .156 \\
\hline Arrhythmia & $183(8.0 \%)$ & $49(6.0 \%)$ & .060 \\
\hline $\begin{array}{l}\text { Peripheral vascular } \\
\text { disease }\end{array}$ & $310(13.6 \%)$ & $143(17.6 \%)$ & .006 \\
\hline \multicolumn{4}{|l|}{ Perioperative factors } \\
\hline $\begin{array}{l}\text { Intraoperative } \\
\text { glucose } \mathrm{mg} / \mathrm{dL} \\
\text { (mean } \pm \mathrm{SD} \text { ) }\end{array}$ & $125.5(19.7)$ & $143.1(29.0)$ & $<.001$ \\
\hline $\begin{array}{l}\text { Postoperative } \\
\text { glucose } \mathrm{mg} / \mathrm{dL} \\
\text { (mean } \pm \mathrm{SD} \text { ) }\end{array}$ & $125.7(24.0)$ & $154.2(46.5)$ & $<.001$ \\
\hline $\begin{array}{l}\text { Arterial grafts (mean } \\
\quad \pm S D)\end{array}$ & $1.39 \pm 0.72$ & $1.40 \pm 0.72$ & .606 \\
\hline $\begin{array}{l}\text { Vein grafts (mean } \pm \\
\text { SD) }\end{array}$ & $1.88 \pm 1.07$ & $1.95 \pm 1.06$ & .105 \\
\hline $\begin{array}{l}\text { Total grafts (mean } \pm \\
\quad \text { SD) }\end{array}$ & $3.27 \pm 1.02$ & $3.36 \pm 1.01$ & .040 \\
\hline $\begin{array}{l}\text { CPB time (min, mean } \\
\pm \mathrm{SD} \text { ) }\end{array}$ & $99.1 \pm 27.9$ & $101.2 \pm 27.2$ & .465 \\
\hline CPB used & $661(29.1 \%)$ & $283(34.7 \%)$ & .002 \\
\hline LITA or BITA used & $2165(95.2 \%)$ & $782(96.1 \%)$ & .291 \\
\hline
\end{tabular}

HbA1c, Hemoglobin A1c; NYHA, New York Heart Association; CCS, Canadian Cardiovascular Society; $S D$, standard deviation; $C P B$, cardiopulmonary bypass; LITA, left internal thoracic artery; BITA, bilateral internal thoracic artery. Intraoperative glucose $=$ postoperative day 0 . Postoperative glucose $=$ postoperative days $1-3$.

of the American Diabetes Association, which recommends a target HbAlc of less than $7 \% .{ }^{17,18}$ Race was modeled as a binary variable (caucasian or non-caucasian), as was New York Heart Association classification (class 4 or non-class 4), Canadian Cardiovascular Society classification (class 4 or non-class 4 ), and chronic lung disease (presence or absence). The outcomes collected for consideration were in-hospital mortality, renal failure, myocardial infarction (MI), cerebrovascular accident (CVA), deep sternal wound infection (DSWI), presence of any infection (superficial or DSWI, pneumonia or septicemia), atrial fibrillation, and postoperative length of stay (LOS).

Missing data were present in race $(\mathrm{n}=83)$, New York Heart Association classification $(\mathrm{n}=35)$, and Canadian Cardiovascular Society classification $(n=38)$. To prevent patients with missing data from being excluded from the analysis, multiple imputation methods described by Rubin ${ }^{19}$ were used to impute missing values. The goal of multiple imputations is not to replace data with plausible values but rather to impute with values that will maintain the covariance structure so that valuable data that are not missing will not be omitted from analysis. Ten separate multiple imputations were generated (using PROC MI, SAS version 9.1, SAS Institute Inc, Cary, NC), and the results were combined (using PROC MIANALYZE) to evaluate all model terms.

Separate multiple logistic regression models (one for each binary outcome) were constructed using HbAlc as the primary variable of interest and adjusting for the other 22 risk factors (Table 1). Of interest was whether $\mathrm{HbA} 1 \mathrm{c}$, modeled as a continuous factor, was predictive of adverse outcomes in the presence of diabetes status and other potential confounders. Adjusted odds ratios (ORs) were reported and measure the additional increase in odds of outcome per unit increase in HbAlc.

For each binary adverse event outcome, 2 models were constructed. The first type of model is an adjusted model (accounting for all of the covariates) and is designed to measure the association between HbA1c apart from the confounders. The second model is unadjusted and designed to measure the "pure" effect of HbA1c without any adjustors. To further evaluate the impact of $\mathrm{HbA} 1 \mathrm{c}$ in explaining each outcome, the discrimination of these models was evaluated using the area under the receiver operating characteristic (AUROC) curve, a measure of discrimination. AUROC is estimated by the c-index, which was used for this study. Discrimination is the model's ability to distinguish between patients who have an event and patients who do not have an event. The AUROC ranges from 0.50 to 1.00 ; values closer to 0.50 indicate poor discrimination, and values closer to 1.00 indicate superior discrimination. Alternatively, AUROC may be thought of as the probability that a randomly selected patient who has an adverse event will have a higher predicted probability of exhibiting the event than a randomly selected unaffected patient. For each outcome and model, the AUROC was computed for the logistic regression model.

Of further interest was to identify the HbA1c threshold value that maximized the AUROC as a binary predictor of adverse outcomes. To evaluate this, HbA1c was constructed as a binary variable with a threshold that was continually shifted by increments of $0.1 \%$ units. Each time the threshold was shifted, the impact of the binary variable was reevaluated in both the unadjusted and adjusted logistic models. The threshold value whose resultant binary predictor maximized the AUROC was recorded for each adverse outcome in the unadjusted and adjusted models. The OR for the unadjusted and adjusted thresholds that maximized the AUROC was also computed and recorded.

LOS was examined with respect to continuous HbA1c using an adjusted general linear model (analysis of covariance approach). Partial tests were performed to test for a non-zero slope for the HbA1c term.

The data were managed and analyzed with SAS version 9.1. All statistical tests were evaluated using a 2-tailed .05 level of significance. Model terms and statistical comparisons were preplanned. The authors had full access to the data and take responsibility for its integrity. All authors have read and agree to the article as written.

\section{Preoperative Definitions}

Definitions for preoperative variables were according to the Society of Thoracic Surgeons National Database and are as follows: diabetes 
indicates a history of diabetes, regardless of duration of disease or need for antidiabetic agents; renal failure indicates whether a patient has a history of renal failure or a creatinine level greater than 2 ; stroke indicates whether a patient has a history of a central neurologic deficit lasting more than 72 hours; left main coronary disease indicates narrowing of the diameter of the lumen by more than $50 \%$ in the left main coronary artery; peripheral vascular disease indicates whether a patient has a history of claudication with exercise or rest, amputation for arterial insufficiency, vascular surgery or percutaneous vascular intervention, or abdominal aortic occlusion or aneurysm; chronic lung disease is determined by forced expiratory volume in 1 second, history of inhaled or oral bronchodilator therapy, use of steroids aimed at treating lung disease, and $\mathrm{PAO}_{2}$ and $\mathrm{PACO}_{2}$ levels; arrhythmia indicates a history of arrhythmia (atrial fibrillation/flutter, ventricular tachycardia/fibrillation, third-degree heart block) that has been documented and treated; hypertension is documented as history of hypertension treated with medication, diet, or exercise, or systolic blood pressure greater than 140 or diastolic blood pressure greater than 90 on 2 separate occasions, or currently receiving antihypertensive medication. Canadian Cardiovascular Society classification was used to define angina and the New York Heart Association criteria (The Criteria Committee) were used to define congestive heart failure.

\section{Postoperative Definitions}

Definitions for postoperative outcomes were also according to the Society of Thoracic Surgeons National Database and are as follows: in-hospital death indicates whether a patient died during the hospitalization in which CABG was performed; renal failure indicates acute renal failure in which serum creatinine was elevated more than 2.0 and 2 times the most recent preoperative creatinine level; $\mathrm{MI}$ indicates a creatine kinase-MB elevation 5 or more times the upper limit of normal and presence of Q waves in 2 or more contiguous ECG leads during the initial 24 postoperative hours, or evolutionary ST-segment changes or new left bundle branch block or the aforementioned criteria after the initial 24 postoperative hours; CVA indicates whether a patient had a central neurologic deficit persisting more than 72 hours (permanent stroke), transient ischemic attack, deficit resolving within 24 hours, or deficit lasting more than 24 hours but less than 72 hours (reversible ischemic neurologic deficit); DSWI indicates whether a patient had an infection involving muscle, bone, or mediastinum requiring operative intervention; atrial fibrillation indicates whether a patient developed new-onset atrial fibrillation in the postoperative setting. In addition to these complications, we included a composite of infection index that included the presence of any sternal wound infection (deep or superficial), pneumonia, or septicemia.

\section{Results}

A total of 3089 of 3555 patients (86.9\%) from April 4, 2002, to June 30,2006 , were analyzed in this analysis (2275 with HbA1c $<7 \%, 814$ with HbA1c $\geq 7 \%$ ). Beginning in 2002, as part of our routine preoperative labs, we obtained preoperative $\mathrm{HbA} 1 \mathrm{c}$ levels in all patients undergoing elective CABG. The lack of uniform adoption of this screening method until July of 2002 accounts for the fact that only $87 \%$ of patients had HbA1c levels obtained during the study period. Preoperative demographics, clinical variables, and perioperative factors comparing patients with $\mathrm{HbA} 1 \mathrm{c}$ greater than or less than 7 are listed in Table 1. Forty-two percent $(516 / 1240)$ of diabetic patients were well-controlled with HbA1c levels less than 7\%. Of note, 4.9\% (90/1849) of patients without a history of diabetes had HbA1c levels of $7 \%$ or greater, suggesting undiagnosed and/or untreated diabetes mellitus. Similarly, $11 \%(90 / 814)$ of patients with $\mathrm{HbA1c}$ levels of $7 \%$ or greater had no history of diabetes. Most patients in this study had multivessel coronary disease, more than $95 \%$ of patients received arterial grafting with the left internal thoracic artery or bilateral internal thoracic artery grafting, and the majority of cases were performed without the use of CPB. The mean glucose values for the day of surgery $(r=0.37)$ and postoperative days 1 to $3(r=0.40)$ were both moderately correlated with preoperative $\mathrm{HbAlc}$ values.

\section{Unadjusted Outcomes}

In-hospital outcomes are summarized by HbA1c subgroup in Table 2. For all patients $(\mathrm{N}=3089)$, the overall incidence of major adverse events (death, CVA, or MI) was low (3.0\%). All outcomes were significantly worse in those patients with $\mathrm{HbA} 1 \mathrm{c}$ levels of $7 \%$ or more, with the exception of postoperative MI and atrial fibrillation. The preoperative method of glucose control for patients with a history of diabetes was also significantly different between groups $(P=.006)$. In diabetic patients with $\mathrm{HbA1c}$ less than $7 \%$, glucose was controlled with diet alone in $20.5 \%$ (106), oral hypoglycemic medications in $58.7 \%$ (303), and subcutaneous insulin in $18.8 \%$ (97). In diabetic patients with $\mathrm{HbA1c}$ of $7 \%$ or more, glucose was controlled with diet alone in $10.5 \%$ (76), oral hypoglycemic medications in $51.5 \%$ (373), and subcutaneous insulin in $37.9 \%$ (275). Patients who were managed with a combination of oral hypoglycemic

TABLE 2. In-hospital outcomes for all patients by HbA1c risk group

\begin{tabular}{|c|c|c|c|}
\hline Outcomes & $\frac{\mathrm{HbA1c}<7.0 \%}{\mathrm{~N}=2275(\%)}$ & $\frac{\mathrm{HbA1c} \geq 7.0 \%}{\mathrm{~N}=814(\%)}$ & $P$ value \\
\hline Death & $18(0.8 \%)$ & $13(1.6 \%)$ & .048 \\
\hline Renal failure & $40(1.8 \%)$ & $40(4.9 \%)$ & $<.001$ \\
\hline $\mathrm{MI}$ & $7(0.3 \%)$ & $4(0.5 \%)$ & .45 \\
\hline CVA & $30(1.3 \%)$ & $23(2.8 \%)$ & .005 \\
\hline DSWI & $10(0.4 \%)$ & $19(2.3 \%)$ & $<.001$ \\
\hline $\begin{array}{l}\text { Composite infection } \\
\text { score }^{a}\end{array}$ & $21(0.9 \%)$ & $26(3.2 \%)$ & $<.001$ \\
\hline Atrial fibrillation & $427(18.8 \%)$ & $122(15.0 \%)$ & .015 \\
\hline $\operatorname{LOS}(\mathrm{d}$, mean $\pm \mathrm{SD})$ & $5.90 \pm 4.47 \mathrm{~d}$ & $6.88 \pm 5.91 \mathrm{~d}$ & $<.001$ \\
\hline
\end{tabular}

$M I$, Myocardial infarction; CVA, cerebrovascular accident; $D S W I$, deep sternal wound infection; $L O S$, length of stay; $S D$, standard deviation. ${ }^{\text {a Infection }}$ is a composite index of any sternal wound infection (deep or superficial), pneumonia, or septicemia. 
TABLE 3. In-hospital outcomes for patients classified by HbA1c level and history of diabetes mellitus

\begin{tabular}{|c|c|c|c|c|c|c|}
\hline \multirow[t]{2}{*}{ Outcomes } & \multicolumn{3}{|c|}{ HbA1c < 7.0\% } & \multicolumn{3}{|c|}{$\mathrm{HbA1c} \geq 7.0 \%$} \\
\hline & No DM & Yes DM & & No DM & Yes DM & \\
\hline Diabetes status & $\mathbf{N}=1759(\%)$ & $\mathbf{N}=\mathbf{5 1 6}(\%)$ & $P$ value & $\mathbf{N}=90(\%)$ & $\mathbf{N}=724(\%)$ & $P$ value \\
\hline Death & $15(0.9 \%)$ & $3(0.6 \%)$ & .54 & $3(3.3 \%)$ & $10(1.4 \%)$ & .16 \\
\hline Renal failure & $26(1.5 \%)$ & $14(2.7 \%)$ & .061 & $2(2.2 \%)$ & $38(5.3 \%)$ & .21 \\
\hline $\mathrm{MI}$ & $6(0.3 \%)$ & $1(0.2 \%)$ & .60 & $0(0)$ & $4(0.6 \%)$ & .48 \\
\hline CVA & $21(1.2 \%)$ & $9(1.7 \%)$ & .34 & $2(2.2 \%)$ & $21(2.9 \%)$ & .71 \\
\hline DSWI & $7(0.4 \%)$ & $3(0.6 \%)$ & .58 & $0(0)$ & $19(2.6 \%)$ & .12 \\
\hline $\begin{array}{l}\text { Composite infection } \\
\text { score }^{\mathrm{a}}\end{array}$ & $12(0.7 \%)$ & $9(1.7 \%)$ & .027 & $1(1.1 \%)$ & $25(3.5 \%)$ & .23 \\
\hline Atrial fibrillation & $319(18.1 \%)$ & $108(20.9 \%)$ & .16 & $13(14.4 \%)$ & $109(15.1 \%)$ & .88 \\
\hline $\operatorname{LOS}(d$, mean $\pm S D)$ & $5.7 \pm 3.9$ & $6.5 \pm 6.0$ & .011 & $6.0 \pm 4.0$ & $7.0 \pm 6.1$ & .043 \\
\hline
\end{tabular}

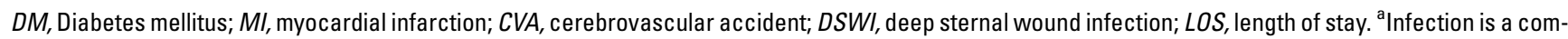
posite index of any sternal wound infection (deep or superficial), pneumonia, or septicemia.

medications and subcutaneous insulin therapy were grouped into those who received subcutaneous insulin. Pre- and postoperative treatment with aspirin, beta-blockers, and statins were not significantly between HbA1c groups. Preoperatively, approximately $77 \%$ of patients received aspirin, $70 \%$ of patients received beta-blockers, and $21 \%$ of patients received statins. Angiotensin-converting enzyme inhibitors were used more often in patients with $\mathrm{HbA} 1 \mathrm{c}$ of $7 \%$ or more $(38.6 \%$ vs $32.3 \%, P=.001)$. Postoperatively, approximately $93 \%$ of patients received aspirin, $93 \%$ of patients received beta-blockers, and $90 \%$ of patients received statins. Angiotensin-converting enzyme inhibitors were used more often in patients with $\mathrm{HbA} 1 \mathrm{c}$ of $7 \%$ or more $(43.8 \%$ vs $32.1 \%, P<.001)$. Of the 18 deaths in patients with HbA1c less than $7 \%, 10$ were cardiac related and 8 were due to various causes (intraoperative [1], multisystem organ failure [2], pulmonary [3], renal failure [1], stroke [1]). Of the 13 deaths in patients with $\mathrm{HbA} 1 \mathrm{c}$ of $7 \%$ or more, 9 were cardiac related. One patient died of complications of stroke, and 3 patients

TABLE 4. In-hospital outcomes among diabetic patients according to $\mathrm{HbA1c}$ level

\begin{tabular}{lcccc}
\hline & HbA1c $<\mathbf{7 . 0 \%}$ & HbA1c $\geq \mathbf{7 . 0} \%$ & \\
\cline { 2 - 2 } & $\mathbf{N}=\mathbf{5 1 6}(\%)$ & $\mathbf{N}=\mathbf{7 2 4}(\%)$ & $\boldsymbol{P}$ value \\
\hline Death & $3(0.6 \%)$ & $10(1.4 \%)$ & .173 \\
Renal failure & $14(2.7 \%)$ & $38(5.3 \%)$ & .028 \\
Myocardial infarction & $1(0.2 \%)$ & $4(0.6 \%)$ & .326 \\
CVA & $9(1.7 \%)$ & $21(2.9 \%)$ & .191 \\
DSWI & $3(0.6 \%)$ & $19(2.6 \%)$ & .007 \\
Infection ${ }^{\text {a }}$ & $9(1.7 \%)$ & $25(3.5 \%)$ & .069 \\
Atrial fibrillation & $108(20.9 \%)$ & $109(15.1 \%)$ & .007 \\
LOS (d, mean \pm SD) & $6.5 \pm 6.0$ & $7.0 \pm 6.1$ & .124 \\
\hline
\end{tabular}

CVA, Cerebrovascular accident; DSWI, deep sternal wound infection; $L O S$, length of stay; $S D$, standard deviation. "Infection is a composite index of any sternal wound infection (deep or superficial), pneumonia, or septicemia. died of pulmonary complications. There were 34 of 1648 patients (2.1\%) with HbA1c less than $7 \%$ and 9 of 540 patients (1.7\%) with $\mathrm{HbAlc}$ of $7 \%$ or greater who were converted from off- to on-pump CABG. There were 4 deaths in the group with $\mathrm{HbA} 1 \mathrm{c}$ less than $7 \%$ and 0 deaths in the group with $\mathrm{HbA} 1 \mathrm{c}$ of $7 \%$ or more.

Table 3 lists adverse event outcomes by HbA1c risk group subdivided into the presence or absence of history of diabetes mellitus. In those patients with $\mathrm{HbA} 1 \mathrm{c}$ less than $7 \%$, there were no statistical differences in morbidity by diabetes status with the exception of a higher risk of the composite of infection in the diabetic patients ( $1.7 \%$ vs $0.7 \%, P=.027)$. There was, however, a significant increase in length of stay in diabetic patients in each $\mathrm{HbA} 1 \mathrm{c}$ group.

Unadjusted outcomes for diabetic patients were also classified according to $\mathrm{HbA1c}$ risk status to evaluate outcomes among diabetic patients with well-controlled diabetes $($ HbA1c $<7 \%)$ versus those with suboptimal control $($ HbA1c $\geq 7 \%)$ (Table 4). The incidence of adverse events was significantly higher for the patients with uncontrolled diabetes (HbA1c $\geq 7 \%$ group), including renal failure $(P=$ $.028)$, DSWI $(P=.007)$, and the composite index of infection (0.069). Although there was a trend toward more mortality in patients with uncontrolled diabetes $(1.4 \%)$ compared with those with controlled diabetes $(0.6 \%)$, this did not reach statistical significance.

Patients were further classified according to their diabetes status without respect to their HbA1c status. Diabetic patients had significantly higher incidences of renal failure, stroke, DSWI, the composite index of infection, and longer LOS (Table 5). There were no significant differences in in-hospital mortality based on diabetes status.

\section{Adjusted Outcomes}

The primary aim of this cohort study was to determine whether HbAlc was an independent risk factor for adverse 
TABLE 5. Outcomes according to diabetes status only, irrespective of HbA1c level

\begin{tabular}{|c|c|c|c|}
\hline \multirow[b]{2}{*}{ Diabetes status } & No DM & Yes DM & \multirow[b]{2}{*}{$P$ value } \\
\hline & $\mathrm{N}=1849(\%)$ & $\mathrm{N}=1240(\%)$ & \\
\hline Death & $18(0.97 \%)$ & $13(1.05 \%)$ & .838 \\
\hline Renal failure & $28(1.5 \%)$ & $52(4.2 \%)$ & $<.001$ \\
\hline Myocardial infarction & $6(0.3 \%)$ & $5(0.4 \%)$ & .719 \\
\hline CVA & $23(1.2 \%)$ & $30(2.4 \%)$ & .014 \\
\hline DSWI & $7(0.4 \%)$ & $22(1.8 \%)$ & $<.001$ \\
\hline Infection $^{a}$ & $13(0.7 \%)$ & $34(2.7 \%)$ & $<.001$ \\
\hline Atrial fibrillation & $332(18.0 \%)$ & $217(17.5 \%)$ & .745 \\
\hline $\operatorname{LOS}(d$, mean $\pm S D)$ & $5.8(3.9 \%)$ & $6.8(6.1 \%)$ & $<.001$ \\
\hline
\end{tabular}

DM, Diabetes mellitus; CVA, cerebrovascular accident; $D S W I$, deep sternal wound infection; $L O S$, length of stay; $S D$, standard deviation. ${ }^{a}$ Infection is a composite index of any sternal wound infection (deep or superficial), pneumonia, or septicemia.

outcomes after CABG. Although HbA1c is organized into risk groups in Tables 2 to 5 for descriptive purposes, it was modeled as a continuous factor in multivariable logistic regression models (Table 6). Higher HbA1c percentage, adjusted for the 22 covariates, was associated with increased incidence of death $(\mathrm{OR}=1.40, P=.019)$, $\mathrm{MI}(\mathrm{OR}=$ $1.55, P=.05)$, and DSWI $(1.38, P=.029)$ for each unit increase in HbA1c. Higher HbA1c was protective of developing atrial fibrillation $(0.89, P=.014)$.

The thresholds that maximize the AUROC when using $\mathrm{HbA1c}$ as a binary variable in unadjusted and adjusted models are listed in Table 7. Patients with HbAlc values of $8.6 \%$ or more have adjusted odds of death 4.41 times higher than patients with values below that threshold. Similar thresholds exist for renal failure $(6.7 \%$, OR $=2.10)$, CVA $(7.6 \%, \mathrm{OR}=2.23)$, and DSWI $(7.8 \%, \mathrm{OR}=5.29)$. Higher $\mathrm{HbA} 1 \mathrm{c}$ is protective of atrial fibrillation $(6.8 \%$, OR $=$ 0.73 ). The model to identify a threshold value for perioperative MI was inestimable (because of small response, $\mathrm{n}=11$ ), so no threshold value could be reliably determined.
TABLE 6. HbA1c adjusted odds ratios for adverse outcomes, measuring the additional risk for every unit increase in HbA1c percentage

\begin{tabular}{lcc}
\hline & \multicolumn{1}{c}{ Continuous HbA1c adjusted } & \\
\cline { 2 - 2 } \multicolumn{1}{c}{ Adverse outcome } & OR (95\% CI) & P value \\
\hline Death & $1.40(1.06-1.86)$ & .019 \\
Renal failure & $1.12(0.94-1.33)$ & .21 \\
Myocardial infarction & $1.55(1.00-2.41)$ & .05 \\
CVA & $1.07(0.86-1.34)$ & .54 \\
DSWI & $1.38(1.03-1.84)$ & .029 \\
Infection $^{\text {a }}$ & $1.18(0.96-1.45)$ & .13 \\
Atrial fibrillation & $0.89(0.80-0.98)$ & .014 \\
Blood products & $0.99(0.92-1.06)$ & .72 \\
\hline
\end{tabular}

$O R$, Odds ratio; CVA, cerebrovascular accident; $D S W I$, deep sternal wound infection. Infection is a composite of any stern wound infection (deep or superficial), pneumonia, or septicemia.

The unadjusted threshold was lower for death $(7.7 \%$, $\mathrm{OR}=6.02$ ). The unadjusted threshold for MI, which had an inestimable adjusted threshold, was determined to be $9.8 \%$, perhaps the weakest estimate because of the small MI event rate.

\section{Discussion}

In the present study, we examined 3089 patients undergoing elective primary CABG. Overall in-hospital mortality in all patients was low $(1.0 \%)$. However, HbA1c levels of $7 \%$ or more in all patients was associated with a significant increase in in-hospital mortality, renal failure, CVA, DSWI, and the composite index of infection compared with patients with $\mathrm{HbA} 1 \mathrm{c}$ levels less than $7 \%$. After adjustment, using a multivariate analysis with $\mathrm{HbA} 1 \mathrm{c}$ as a continuous variable, the risk of in-hospital death was significantly higher $(P<.001)$ with an OR of 1.40. This translates into a $40 \%$ increase in odds of death for each unit increase in HbA1c. By using a receiver operating characteristic curve analysis, which maximizes the area under the curve and identifies the HbA1c threshold

TABLE 7. Threshold values that maximize the area under the receiver operating characteristic curve in adjusted and unadjusted models

\begin{tabular}{lccccccc}
\hline & \multicolumn{3}{c}{ Adjusted ROC estimates } & & \multicolumn{3}{c}{ Unadjusted ROC estimates } \\
\cline { 2 - 4 } \cline { 6 - 7 } Adverse outcome & Threshold value & AUROC & OR & & Threshold value & AUROC & OR \\
\hline Death & 8.6 & 0.906 & $4.41(1.44-13.42)$ & & 7.7 & 0.590 & $6.02(2.16-16.80)$ \\
Renal failure & 6.7 & 0.792 & $2.10(1.16-3.30)$ & & 6.7 & 0.633 & $3.03(1.94-4.76)$ \\
MI & Inestimable model & - & - & 9.8 & & 0.607 & $6.07(1.60-23.09)$ \\
CVA & 7.6 & 0.791 & $2.23(1.06-4.70)$ & & 7.2 & 0.590 & $2.31(1.33-4.02)$ \\
DSWI & 7.8 & 0.801 & $5.29(2.00-14.00)$ & & 7.7 & 0.725 & $7.90(3.71-16.83)$ \\
Infection $^{\text {a }}$ & 7.5 & 0.761 & $1.98(0.96-4.09)$ & & 6.9 & 0.650 & $3.58(2.00-6.42)$ \\
Atrial fibrillation & 6.8 & 0.687 & $0.73(0.55-0.96)$ & & 7.1 & 0.530 & $0.72(0.57-0.90)$ \\
\hline
\end{tabular}

MI, Myocardial infarction; CVA, cerebrovascular accident; DSWI, deep sternal wound infection; $R O C$, receiver operating characteristic; $A U R O C$, area under

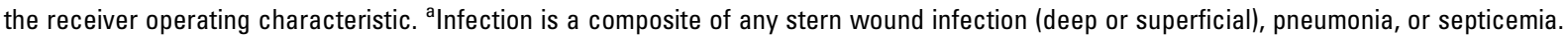


most likely to separate outcome dichotomies, the adjusted (unadjusted) threshold value for HbAlc was 8.6 (7.7) for in-hospital death with an adjusted OR of 4.4 and an unadjusted OR of 6.0. This translates to a 4-fold increase in the risk of in-hospital death for patients with HbA1c more than $8.6 \%$.

In this study, 1240 of 3089 patients (40\%) presented with a history of diabetes mellitus. Even within the Emory Healthcare System, we have seen an increase in diabetic patients referred for surgical revascularization. In our previous report, ${ }^{2}$ only 19\% of patients undergoing CABG from 1978 to 1993 had a history of diabetes mellitus. According to the Bypass Angioplasty Revascularization Investigation, patients with multivessel disease had improved survival when treated with an initial strategy of CABG versus percutaneous intervention. ${ }^{20}$ Therefore, we expect a larger proportion of patients with multivessel coronary disease to be referred for surgical revascularization.

Recent studies comparing diabetic and nondiabetic patients after CABG have yielded conflicting results regarding in-hospital and short-term outcomes. Calafiore and colleagues ${ }^{6}$ showed that diabetes was an independent risk factor for early cardiac death only and not for all-cause mortality. Rajakaruna and associates ${ }^{5}$ found in a risk-adjusted analysis that diabetic patients had mortality outcomes comparable to those of nondiabetic patients. Kubal and coworkers ${ }^{7}$ showed that insulin-dependent diabetic patients had an increased risk of postoperative morbidity, but that just the history of diabetes was not associated with increased mortality. Other studies have refuted these claims, including work by Carson and colleagues, ${ }^{3}$ Thourani and colleagues, ${ }^{2}$ and Woods and colleagues. ${ }^{4}$ In several of these studies, the mode of glycemic control was used to stratify diabetic patients into those with glucose controlled with oral hypoglycemic medications versus insulin therapy, ${ }^{2,7}$ as well as those with other complications of diabetes, such as renal failure and peripheral vascular disease. ${ }^{21}$ The largest study to date by Carson and colleagues $^{3}$ examined outcomes in 41,663 diabetic patients compared with 105,123 nondiabetic patients and found that patients with diabetes had a $23 \%$ to $37 \%$ increase in 30-day mortality and in-hospital morbidity compared with patients without diabetes. Some of these differences may be explained by how diabetic patients were defined. For example, exclusion of those with diet-controlled diabetes or exclusion of those without a preoperative history of diabetes (which may have occurred in retrospective analyses) may alter the comparisons between groups.

The practice changes in the past decade compared with earlier reported studies may also have affected the lack of a mortality difference between diabetic and nondiabetic patients. Specifically, the routine use of the left internal thoracic artery, ${ }^{22}$ improvements in anesthesia and critical care, the use of off-pump CABG techniques, perioperative insulin infusion, ${ }^{10-15}$ and improved secondary prevention protocols, ${ }^{23}$ including antiplatelet medication, lipid-lowering regimens, and preoperative glucose modulation with insulin and oral hypoglycemic regimens, may have collectively improved outcomes in diabetic patients undergoing CABG. These differences may make interpretation of older studies ${ }^{24-28}$ less reliable compared with current reports. In the present study, we sought to determine whether these outcome differences could be explained by preoperative glycemic control (as measured by $\mathrm{HbAlc}$ ) rather than the mere history of diabetes mellitus.

One of the most dramatic improvements in outcomes among diabetic patients has been the implementation of tight perioperative glucose control. Furnary and colleagues ${ }^{10,14,15}$ reported dramatic reductions in mortality and DSWI among diabetic patients (comparable to nondiabetic patients) managed with a continuous insulin infusion initiated intraoperatively and maintained through the first 2 postoperative days. The authors attributed these improvements to enhanced myocardial glycometabolic function associated with euglycemia achieved by continuous insulin infusion. We aimed to regulate glucose levels between 80 and $110 \mathrm{mg} / \mathrm{dL}$ in all patients in the operating room and in the intensive care unit using a continuous insulin infusion. Once patients were transferred to the telemetry unit, management was variable and dependent on their control in the intensive care unit. This included the use of continuous insulin infusion, scheduled subcutaneous insulin therapy, and a sliding scale. Although patients with preoperative HbA1c 7\% or more had significantly higher intra- and postoperative mean glucose levels compared with patients with $\mathrm{HbA1c}$ less than $7 \%$, the mean glucose levels in patients with $\mathrm{HbA} 1 \mathrm{c} 7 \%$ or more (day 0: $143 \mathrm{mg} / \mathrm{dL}$, days 1-3: $154 \mathrm{mg} / \mathrm{dL}$ ) were comparable to those of diabetic patients in other studies. ${ }^{10-15}$ Although we did not achieve the desired target blood glucose levels in the operating room and intensive care unit, perioperative glucose control was reasonable. In addition, preoperative $\mathrm{HbA1c}$ level still emerged as an independent predictor of mortality and DSWI after CABG in the multivariate analysis, even after adjusting for mean glucose levels on the day of surgery and the first 3 postoperative days. This is in contrast with the findings of Furnary and $\mathrm{Wu},{ }^{10}$ which did not identify $\mathrm{HbA1c}$ as a risk factor. Therefore, we agree with previous authors that strict intraoperative and postoperative glucose control is imperative to minimize both postoperative morbidity and mortality after CABG. However, it is plausible from these data that $\mathrm{HbA} 1 \mathrm{c}$ is also an independent predictor of postoperative adverse events that is not entirely due to intraand postoperative glucose control. This is most likely explained by the associated comorbidities that patients with long-standing diabetes present with before $\mathrm{CABG}$, including renal insufficiency, cerebrovascular disease, hypertension, and more advanced coronary artery disease as seen in the preoperative comorbidities in our patients with elevated $\mathrm{HbAlc}$. In addition to predicting adverse events, it may be more 
difficult to achieve intra- and postoperative euglycemia in patients with elevated HbA1c. Therefore, more aggressive glucose management should be used in these patients in the perioperative period.

HbA1c reflects a patient's glucose control during the preceding 3 to 4 months. Current practice guidelines according to the American Diabetes Association recommend routine HbA1c screening of diabetic patients as frequently as every 3 months in patients with elevated A1c levels ( $\geq 7 \%$ ) or in patients with medication adjustments to ensure an adequate treatment regimen. ${ }^{13}$ Current recommendations suggest that patients with diabetes aim to achieve HbA1c levels of at least less than $7 \% .{ }^{17,18,29,30} \mathrm{We}$ chose to order HbA1c for all patients starting in 2002 (even those without a history of diabetes) to determine whether this approach would identify patients who had uncontrolled hyperglycemia without a previous diagnosis of diabetes mellitus. As seen in Table 3, 90 patients in this study without a previous diagnosis of diabetes had preoperative $\mathrm{HbA} 1 \mathrm{c}$ levels of $7 \%$ or greater. This prompted confirmatory diagnosis in the postoperative setting and endocrinology consultation for management of diabetes.

To our knowledge, this is one of the few studies that assessed whether $\mathrm{HbA1c}$, the standard measure to assess long-term glucose control, is a potential risk factor for adverse outcomes in patients undergoing CABG. ${ }^{10}$ It is possible that a preoperative $\mathrm{HbA1c}$ level may provide more accurate prognostic information about outcomes after CABG compared with a diabetes status alone. In patients with diabetes but with good long-term preoperative glycemic control (HbA1c $<7 \%$ ), outcomes (with the exception of the composite index of infection) were comparable to those of patients without a diagnosis of diabetes and with $\mathrm{HbAlc}$ less than 7\%. In addition, we implemented continuous insulin infusion to achieve glucose control on the day of surgery and in the intensive care unit. This may have accounted for the lack of a mortality difference between diabetic patients and nondiabetic patients in the unadjusted analysis. Because other studies evaluating the impact of diabetes on morbidity and mortality after CABG have resulted in different conclusions, we hypothesize that some of these differences may in part be due to the lack of a standardized measure (HbA1c) to adjust for significant differences in long-term preoperative glycemic control. This would apply to all diabetic patients, irrespective of the mode of therapy used for glucose control, suggesting that some patients should begin more intense glucose control with insulin therapy before CABG. Moreover, in patients with $\mathrm{HbA} 1 \mathrm{c}$ greater than $8.7 \%$ and not requiring an urgent or emergency $\mathrm{CABG}$, consideration should be given to maximizing blood glucose control before surgery to minimize morbidity and mortality.

\section{Limitations}

Our study has the inherent biases of any retrospective review of a prospectively collected database. Although we con- trolled for multiple variables, there may still be confounding factors that may have altered the results. For instance, even though we controlled for perioperative glucose values in the multivariable analysis, it is possible that better glucose control in patients with an elevated HbA1c may have reduced adverse events in this group. Furthermore, although we endeavored to only use a limited set of confounders, for some outcomes with rare event rates (MI), the number of covariates might be disproportionately higher than the "rule of 10" (at least 10 events for every covariate added to a model) would suggest. Our study population may be different from those of other studies with regard to surgical approach and perioperative care. Approximately $70 \%$ of patients underwent CABG without the use of CPB. All patients were treated with a strict perioperative insulin infusion and then transitioned to subcutaneous insulin to target blood glucose less than $150 \mathrm{mg} / \mathrm{dL}$. Although we used a multivariate analysis model, we did not use propensity matching in our subgroup analyses. The goal of propensity scoring is to identify whether some intervention is associated with an outcome while working to balance these intervention groups with respect to their other preoperative characteristics. The problem with propensity scoring in our present study is that there is no intervention; HbAlc is a pure preoperative risk factor and not any type of intervention. Furthermore, we did not determine whether insulin-dependent patients had worse outcomes compared with those with diabetes controlled with diet or oral hypoglycemic medications, as has been done in other studies. In this study, only patients who reported a history of diabetes preoperatively were classified as "diabetic." In this retrospective analysis, patients with no known history of diabetes were classified as "nondiabetic" even if they were determined to be diabetic in the postoperative period. In these patients, their diagnosis would have been overlooked without a preoperative HbA1c level.

\section{Conclusions}

This study showed that $\mathrm{HbA} 1 \mathrm{c}$ is a powerful predictor of inhospital death and morbidity. HbA1c may be a more accurate predictor of outcomes than merely a diagnosis of diabetes, with the added benefit of quantification of diabetes as a risk factor. This simple laboratory test drawn preoperatively may provide the clinician with a more accurate risk profile and provide additional prognostic information when discussing morbidity and mortality risks with patients and their families. Whether this warrants practice changes in patients with poorly controlled or newly diagnosed diabetes will be the subject of future investigation.

\section{References}

1. Kannel WB, McGee DL. Diabetes and cardiovascular risk factors: the Framingham Study. Circulation. 1979;59:8-13.

2. Thourani VH, Weintraub WS, Stein B, Gebhart SSP, Craver JM, Jones EL, et al. Influence of diabetes mellitus on early and late outcomes 
after coronary artery bypass grafting. Ann Thorac Surg. 1999;67: 1045-52.

3. Carson JL, Scholz PM, Chen AY, Petersen ED, Gold J, Schneider SH. Diabetes mellitus increases short-term mortality and morbidity in patients undergoing coronary artery bypass graft surgery. J Am Coll Cardiol. 2002;40:418-23.

4. Woods SE, Smith JM, Sohail S, Sarah A, Engle A. The influence of type 2 diabetes mellitus in patients undergoing coronary artery bypass graft surgery. Chest. 2004;126:1789-95.

5. Rajakaruna C, Rogers CA, Suranimala C, Angelini GD, Ascione R. The effect of diabetes mellitus on patients undergoing coronary surgery: a risk adjusted analysis. J Thorac Cardiovasc Surg. 2006;132:802-10.

6. Calafiore AM, Di Mauro M, Di Giammarco G, Contini M, Vitolla G, Iaco AL, et al. Effect of diabetes on early and late survival after isolated first coronary bypass surgery in multivessel disease. $J$ Thorac Cardiovasc Surg. 2003;125:144-54.

7. Kubal C, Srinivasan AK, Grayson AD, Fabri BM, Chalmers JAC. Effect of risk-adjusted diabetes on mortality and morbidity after coronary artery bypass surgery. Ann Thorac Surg. 2005;79:1570-6.

8. Whang W, Bigger JT. Diabetes and outcomes of coronary artery bypass graft surgery in patients with severe left ventricular dysfunction: results from the CABG Patch Trial Database. J Am Coll Cardiol. 2000;36: 1166-72.

9. Szabo Z, Hakanson E, Svedjeholm R. Early postoperative outcome and medium-term survival in 540 diabetic and 2239 nondiabetic patients undergoing coronary artery bypass grafting. Ann Thorac Surg. 2002;74: 712-9.

10. Furnary AP, Wu Y. Eliminating the diabetic disadvantage: the Portland diabetic project. Semin Thorac Cardiovasc Surg. 2006;18:302-8.

11. Lazar HL, Chipkin SR, Fitzgerald CA, Bao Y, Cabral H, Apstein CS. Tight glycemic control in diabetic coronary artery bypass graft patients improves perioperative outcomes and decreases recurrent ischemic events. Circulation. 2004;109:1497-502.

12. Carr JM, Sellke FW, Fey M, Doyle MJ, Krempin JA, de la Torre R, et al. Implementing tight glucose control after coronary artery bypass surgery. Ann Thorac Surg. 2005;80:902-9.

13. Lazar HL, Chipkin S, Philippides G, Bao Y, Apstein C. Glucose-insulinpotassium solutions improve outcomes in diabetics who have coronary artery operations. Ann Thorac Surg. 2000;70:145-50.

14. Furnary AP, Gao G, Grunkemeier GL, Wu Y, Zerr KJ, Bookin SO, et al. Continuous insulin infusion reduces mortality in patients with diabetes undergoing coronary artery bypass grafting. $J$ Thorac Cardiovasc Surg. 2003; 125:1007-21.

15. Furnary AP, Zerr KJ, Grunkemeier GL, Starr A. Continuous intravenous insulin infusion reduces the incidence of deep sternal wound infection in diabetic patients after cardiac surgical procedures. Ann Thorac Surg. 1999;67:352-62.

16. American Diabetes Association. Standards of medical care in diabetes. Diabetes Care. 2005;28:S4-36.

17. The Diabetes Control and Complications Trial Research Group. The effect of intensive treatment of diabetes on the development and progression of long-term complications in insulin-dependent diabetes mellitus. N Engl J Med. 1993;329:977-86.

18. UK Prospective Diabetes Study (UKPDS) group. Intensive blood-glucose control with sulphonylureas or insulin compared with conventional treatment and risk of complications in patients with type 2 diabetes (UKPDS 33). Lancet. 1998;352:837-53.

19. Rubin DB. Inference and missing data. Biometrika. 1976;53:581-92.

20. The Bypass Angioplasty Revascularization Investigation (BARI) Investigators. Influence of diabetes on 5-year mortality and morbidity in a randomized trial comparing CABG and PTCA in patients with multivessel disease. Circulation. 1997;96:1761-9.

21. Leavitt BJ, Sheppard L, Maloney C, Clough RA, Braxton JH, Charlesworth DC, et al. Effect of diabetes and associated conditions on long-term survival after coronary artery bypass graft surgery. Circulation. 2004;110:41-4.

22. Hirotani T, Kameda T, Kumamoto T, Shirota S, Yamano M. Effects of coronary artery bypass grafting using internal mammary arteries for diabetic patients. J Am Coll Cardiol. 1999;34:532-8.
23. Bradshaw PJ, Jamrozik K, Gilfillan I, Thompson PL. Preventing recurrent events long term after coronary artery bypass graft: suboptimal use of medications in a population study. Am Heart J. 2004;147:1047-53.

24. Lawrie GM, Morris GC Jr, Glaeser DH. Influence of diabetes mellitus on the results of coronary bypass surgery: follow-up of 212 diabetic patients ten to fifteen years after surgery. JAMA. 1986;256:2967-71.

25. Herlitz J, Wognsen GB, Emanuelsson H, Haglid M, Karlson BW, Karlsson T, et al. Mortality and morbidity in diabetic and nondiabetic patients during a 2-year period after coronary artery bypass grafting. Diabetes Care. 1996;30:71-5.

26. Salomon NW, Page US, Okies JE, Stephens J, Krause AH, Bigelow JC. Diabetes mellitus and coronary artery bypass: short-term risk and longterm prognosis. J Thorac Cardiovasc Surg. 1983;85:264-71.

27. Clement R, Rousou JA, Engelman RM, Breyer RH. Perioperative morbidity in diabetics requiring coronary artery bypass surgery. Ann Thorac Surg. 1988;46:321-3.

28. Fietsam R Jr, Bassett J, Glover JL. Complications of coronary artery surgery in diabetic patients. Am Surg. 1991;57:551-7.

29. Saudek CD, Derr RL, Kalyani RR. Assessing glycemia in diabetes using self-monitoring blood glucose and hemoglobin A1c. JAMA. 2006;295: 1688-97.

30. The ACE/ADA Task Force on Inpatient Diabetes. American College of Endocrinology and American Diabetes Association Consensus Statement on inpatient diabetes and glycemic control. Diabetes Care. 2006; 29:1955-62.

\section{Discussion}

Dr Harold L. Lazar (Boston, Mass). In their study, Dr Halkos and colleagues found that an elevated preoperative $\mathrm{HbA} 1 \mathrm{c}$ level was associated with a significant increase in mortality and morbidity. This is not surprising because patients with an elevated HbAlc had a higher incidence of preoperative renal failure, strokes, peripheral vascular disease, and diffuse coronary artery disease, which required more grafts. All of these factors are independently known to be predictive of adverse outcomes after bypass surgery.

My first question is whether an elevated HbAlc is a marker of advanced diabetic end-organ disease or does it really represent poor intraoperative glycemic control? Did you actually measure the perioperative glucose values between the high and low HbA1c groups to see whether or not your protocols actually achieved tight glycemic control in the elevated HbAlc group, and did the patients with elevated HbA1c levels who had lower perioperative glucose values have decreased morbidity and mortality?

Dr Halkos. Let me first start by addressing the question regarding tight intraoperative glycemic control. We did not collect those data because they were not accessible to us regarding the blood glucose levels in the operating room and intensive care unit setting. However, all of our patients undergoing primary elective CABG are maintained in the same fashion with regard to the regimen in which their blood glucose is controlled both in the operating room and in the intensive care unit, as well as how they are transitioned on the floor.

Dr Lazar. I think that may be important data because we have found that it is more difficult to achieve tighter glycemic control in patients with elevated HbA1c even though there is a set protocol. I may actually answer your first clinical implication as to whether or not these patients can be delayed. Perhaps these patients may have to have more vigorous intraoperative control of their glucose levels.

My second question is, were the adverse effects of an elevated HbA1c related to the type of diabetes? In other words, did insulindependent diabetic patients have worse outcomes compared with patients treated with oral agents or diet alone? 
Dr Halkos. Let me answer that with regard to a previous question that you asked regarding patients with elevated HbA1c: Are the intraoperative and perioperative glucose levels or the comorbidities associated with poorly controlled diabetes? We believe that it may very well be the comorbidities associated with poorly controlled diabetes, which is reflected by an elevated HbA1c. With regard to the last question, rephrase that last part for me.

Dr Lazar. Did insulin diabetic patients have worse outcomes than those with oral agents or diet alone?

Dr Halkos. We did not look into the subgroupings of how diabetes was controlled in patients with regard to their method of control. In our study, approximately $20 \%$ of patients were receiving insulin therapy, and then $50 \%$ were receiving oral hypoglycemic medications or a combination thereof. So part of the reason for not looking at the data in that fashion was the combination thereof was not well defined in our database.

Dr Lazar. Patients in the elevated HbA1c group had significantly higher mortality. What were the differences in the cause of death between the groups? Were the patients with elevated $\mathrm{HbA} 1 \mathrm{c}$ more likely to die of cardiac causes or was it more related to their increased incidence of renal fairly, strokes, and peripheral vascular disease?

Dr Halkos. With regard to the cause of death, we really didn't look into the cause with regard to their cause of mortality other than report the associated morbidities.

Dr Lazar. In your study, $70 \%$ of patients underwent off-pump surgery, yet patients with an elevated HbA1c were more likely to undergo on-pump surgery. What was the crossover rate from the offpump to the on-pump groups in this study, and were the patients with an elevated $\mathrm{HbA1c}$ who crossed over more likely to have an adverse outcome? I must say these data were derived from the article, which you were kind enough to send to me in advance.

Dr Halkos. Yes, sir. I don't know the exact crossover rate, but I know that during the period of the study it was very low, with a $2 \%$ crossover. Patients were categorized according to whether they underwent on- or off-pump surgery according to the ultimate procedure that was used. So that is a piece of data that can be misleading. We believe that even though slightly more patients underwent on-pump surgery in the higher A1c subgroup, that was only approximately $4 \%$ to $5 \%$ higher than the A1c group $(<7 \%)$.

Dr Lazar. Recent studies have shown that patients receiving angiotensin-converting enzyme inhibitors, beta-blockers, and statins in the perioperative period have decreased morbidity and mortality. Can you tell us what percentage of patients in the study received these medications and was there a difference in the use of these medications between the HbA1c groups?

Dr Halkos. Again, almost all of our patients are managed in the same way: They receive aspirin preoperatively, angiotensin-converting enzyme inhibitors are discontinued preoperatively in almost all patients, and they receive beta-blocker therapy.

Dr Lazar. One final question, again from the article. Your data implied that elevated $\mathrm{HbA} 1 \mathrm{c}$ levels were actually protective from developing atrial fibrillation. In other words, people with elevated HbA1c actually had a lower incidence of atrial fibrillation. Can you explain that and give us an idea of what the mechanism was?

Dr Halkos. We reported all of these outcomes, and in this study, which was not presented in the slides, there was a $30 \%$ lower incidence of atrial fibrillation in patients with an elevated A1c, but this was statistically significant, and we do not have a mechanism to explain why that difference existed. 